\title{
Blocking Soluble Tumor Necrosis Factor Signaling with Dominant-Negative Tumor Necrosis Factor Inhibitor Attenuates Loss of Dopaminergic Neurons in Models of Parkinson's Disease
}

\author{
Melissa K. McCoy, ${ }^{1}$ Terina N. Martinez, ${ }^{1}$ Kelly A. Ruhn, ${ }^{1}$ David E. Szymkowski, ${ }^{4}$ Christine G. Smith, ${ }^{2}$ \\ Barry R. Botterman, ${ }^{3}$ Keith E. Tansey, ${ }^{2}$ and Malú G. Tansey ${ }^{1}$ \\ Departments of ${ }^{1}$ Physiology, ${ }^{2}$ Neurology, and ${ }^{3}$ Cell Biology, University of Texas Southwestern Medical Center, Dallas, Texas 75390 , and ${ }^{4}$ Xencor, Inc., \\ Monrovia, California 91016
}

\begin{abstract}
The mechanisms that trigger or contribute to loss of dopaminergic (DA) neurons in Parkinson's disease (PD) remain unclear and controversial. Elevated levels of tumor necrosis factor (TNF) in CSF and postmortem brains of PD patients and animal models of PD implicate this proinflammatory cytokine in the pathophysiology of the disease; but a role for TNF in mediating loss of DA neurons in PD has not been clearly demonstrated. Here, we report that neutralization of soluble TNF (solTNF) in vivo with the engineered dominantnegative TNF compound XENP345 (a PEGylated version of the TNF variant A145R/I97T) reduced by 50\% the retrograde nigral degeneration induced by a striatal injection of the oxidative neurotoxin 6-hydroxydopamine (6-OHDA). XENP345 was neuroprotective only when infused into the nigra, not the striatum. XENP345/6-0HDA rats displayed attenuated amphetamine-induced rotational behavior, indicating preservation of striatal dopamine levels. Similar protective effects were observed with chronic in vivo coinfusion of XENP345 with bacterial lipopolysaccharide (LPS) into the substantia nigra, confirming a role for solTNF-dependent neuroinflammation in nigral degeneration. In embryonic rat midbrain neuron/glia cell cultures exposed to LPS, even delayed administration of XENP345 prevented selective degeneration of DA neurons despite sustained microglia activation and secretion of solTNF. XENP345 also attenuated 6-OHDAinduced DA neuron toxicity in vitro. Collectively, our data demonstrate a role for TNF in vitro and in vivo in two models of PD, and raise the possibility that delaying the progressive degeneration of the nigrostriatal pathway in humans is therapeutically feasible with agents capable of blocking solTNF in early stages of PD.
\end{abstract}

Key words: neuroinflammation; TNF; neuronal apoptosis; degeneration; microglia; Parkinson's disease

\section{Introduction}

Parkinson's disease (PD) is the second most prevalent neurodegenerative disease in the United States with a 5\% incidence in individuals over 65 (Moore et al., 2005). Its clinical manifestations result from selective loss of dopaminergic (DA) neurons in the ventral mesencephalon substantia nigra pars compacta $(\mathrm{SNpc})$, with a resulting decrease in striatal dopamine. The critical molecular mediators and mechanisms that elicit death of nigral DA neurons have yet to be identified; but a wealth of studies implicate microglia and inflammatory processes in the patho-

Received Dec. 30, 2005; revised Aug. 3, 2006; accepted Aug. 4, 2006.

This work was supported by a grant from The Michael J. Fox Foundation for Parkinson's Research. We thank Drs. Mark West, Steven Evans, Lutz Slomianka, and Geoff Greene of MicroBrightField for guidance and advice on stereological analyses; Don Copper in the Department of Psychiatry for allowing us access to his MicroBrightField StereoInvestigator equipment; and Joyce Repa and members of the Tansey laboratory, especially Tamy Frank-Cannon for critical discussions and review of this manuscript and Sabrina Martinez-Anz for technical support in the early days.

Correspondence should be addressed to Malú $G$. Tansey, Department of Physiology, The University of Texas Southwestern Medical Center, 5323 Harry Hines Boulevard, Dallas, TX 75390-9040. E-mail: malu.tansey@ utsouthwestern.edu.

DOI:10.1523/JNEUROSCI.1504-06.2006

Copyright $\odot 2006$ Society for Neuroscience $\quad$ 0270-6474/06/269365-11\$15.00/0 physiology of PD (McGeer et al., 1988; Vawter et al., 1996; Hald and Lotharius, 2005; Hirsch et al., 2005), and chronic use of nonsteroidal anti-inflammatory drugs can lower risks for development of PD in humans by 46\% (Chen et al., 2003).

CSF and postmortem brains of PD patients display elevated levels of the proinflammatory cytokine tumor necrosis factor (TNF) as do animals treated with the dopaminergic neurotoxins 1-methyl-4-phenyl-1,2,3,6-tetrahydropyridine (MPTP) and 6-hydroxydopamine (6-OHDA) used to model nigral degeneration in nonhuman primates and rodents (Boka et al., 1994; Hunot et al., 1999; Mogi et al., 2000; Sriram et al., 2002; Barcia et al., 2005; Nagatsu and Sawada, 2005). TNF is synthesized as a type II transmembrane trimeric protein cleaved by the TACE (TNF- $\alpha$ converting enzyme) metalloprotease to a soluble form (Aggarwal et al., 2000; MacEwan, 2002); both forms are biologically active, but their relative roles in mediating DA neuron survival are unknown. Soluble TNF (solTNF) transduces inflammatory stimuli through the canonical death receptor TNF receptor 1 (TNFR1) (Tartaglia et al., 1993), which is highly expressed in nigrostriatal DA neurons, rendering them vulnerable to TNF-induced toxicity (Aloe and Fiore, 1997; McGuire et al., 2001; Gayle et al., 2002; 
Carvey et al., 2005). The role of transmembrane (tm)TNF is less well understood, but it can mediate prosurvival effects through TNFR2 in cortical (Marchetti et al., 2004) and hippocampal (Heldmann et al., 2005) neurons.

We hypothesized that solTNF is a major mediator of neurotoxic mechanisms contributing to degeneration of nigral DA neurons in vivo; therefore blocking its activity should yield neuroprotection in animal models of $\mathrm{PD}$. To test our hypothesis, we used TNF variants (XENP345) engineered to disrupt binding of the solTNF trimer to its receptors by forming dominant-negative TNF (DN-TNF) heterotrimers that eliminate solTNF homotrimers, and thus inhibit its signaling (Steed et al., 2003). To elucidate the cellular mechanisms by which TNF promotes DA neuron death, we used rat embryonic ventral mesencephalon (EVM) neuron/glia cultures. Our findings that solTNF, but not tmTNF, contributes significantly to the progressive loss of DA neurons induced by bacterial and oxidative neurotoxins in cellular and animal models of PD are relevant for the design and testing of novel therapeutic strategies for PD.

\section{Materials and Methods}

Reagents. Rabbit anti-tyrosine hydroxylase (TH), guinea pig anti-GABA, mouse anti-microtubule-associated protein $2 \mathrm{~b}$ (MAP2b), and mouse anti-neuron-specific nuclear protein (NeuN) antibodies were obtained from Chemicon (Temecula, CA). Mouse anti-rat complement 3 receptor (C3R) antibody Ox-42 was obtained from Santa Cruz Biotechnology (Santa Cruz, CA), and mouse anti-CD45 antibody was obtained from Serotec (Indianapolis, IN). FITC-conjugated isolectin-B4, lipopolysaccharide (LPS) (from Escherichia coli 0111:B4; lot no. 114K4133; $1.5 \times 10^{6}$ $\mathrm{EU} / \mathrm{mg}$ ), 6-OHDA, poly-D-lysine, and D-amphetamine were obtained from Sigma (St. Louis, MO), and a single stock of each was used for all experiments. Cell culture reagents were purchased from Invitrogen (Carlsbad, CA). Laminin was obtained from BD Biosciences (San Jose, CA). The recombinant dominant-negative TNF XENP345, a PEGylated version of the TNF variant A145R/I97T (Steed et al., 2003), was bacterially produced and formulated by Xencor, Inc., to contain $<0.1 \mathrm{EU} / \mathrm{ml}$. Recombinant mouse TNF was obtained from R \& D Systems (Minneapolis, MN). Antibodies for quantitative TNF ELISA were obtained from Biosource/Invitrogen (Carlsbad, CA). Osmotic pumps were purchased from Alzet (Cupertino, CA), cannulas and tubing from Plastics One (Roanoke, VA). All other reagents were obtained from Sigma.

Animal studies. Young adult and timed-pregnant Sprague Dawley SASCO and CDF/Fischer 344 rats were purchased from Charles River Laboratories (Wilmington, MA) and housed in pathogen-free climatecontrolled facilities at the Animal Resources Center at University of Texas Southwestern Medical Center. All animal studies were approved by the Institutional Animal Care and Use Committee at University of Texas Southwestern Medical Center at Dallas.

Intrastriatal 6-OHDA injection and XENP345 infusions. Young adult female Sprague Dawley SASCO rats $(200-225 \mathrm{~g})(n=6$ per group; total of 30$)$ were anesthetized with halothane $(2 \%)$ and placed in a stereotaxic frame. Their eyes were protected with ophthalmic ointment, and body temperature was monitored with a rectal probe and maintained with radiant heat under feedback control. The scalp was prepped under sterile conditions, and the skull was exposed and incised. We chose a previously published regimen of 6-OHDA to induce a mild-to-moderate retrograde lesion in the nigrostriatal pathway (Kirik et al., 1998). Burr holes were drilled to permit unilateral injection of $20 \mu \mathrm{g}$ of 6-OHDA ( $4 \mu \mathrm{l}$ of 5 $\mu \mathrm{g} / \mu \mathrm{l})$ at a rate of $1 \mu \mathrm{l} / \mathrm{min}$ into the striatum on the right hemisphere (stereotaxic coordinates: anteroposterior (AP), $-1.2 \mathrm{~mm}$ from bregma; mediolateral (ML), $-3.9 \mathrm{~mm}$; and dorsoventral (DV), $-5 \mathrm{~mm}$ below surface of dura) (Paxinos et al., 1985). Cannulas (gauge 28; Plastics One) connected via polyethylene tubing to a subcutaneously implanted osmotic minipump (Alzet 2002) preloaded with vehicle (sterile saline) or the treatment agent XENP345 $\left(0.08 \mathrm{mg} \cdot \mathrm{kg}^{-1} \cdot \mathrm{d}^{-1}\right)$ were then stereotaxically inserted through the burr holes into the same site as the 6-OHDA lesion or into an area just above the substantia nigra pars com- pacta (stereotaxic coordinates from bregma: AP, $-4.8 \mathrm{~mm}$ from bregma; $\mathrm{ML},-1.7 \mathrm{~mm}$; and DV,$-8 \mathrm{~mm}$ below surface of dura) through another burr hole and were left in position for 3 weeks. Cannulas were secured to the skull with surgical glue (Plastics One).

Intranigral LPS or LPS/XENP345 infusions. The low-dose chronic LPS infusion model published previously was used to induce selective, delayed, and progressive death of DA neurons in vivo (Gao et al., 2002). LPS $(5 \mathrm{ng} / \mathrm{h}$ ) was unilaterally infused for 2 weeks via a 28 gauge cannula into the SNpc (coordinates from bregma: AP, $-4.8 \mathrm{~mm}$; ML, $-1.7 \mathrm{~mm}$; and DV , $-8 \mathrm{~mm}$ ) (Paxinos et al., 1985) of young adult male CDF rats (200$240 \mathrm{~g}$ ) ( $n=6$ per group; three sets of experiments) under the same surgical procedures described above. Cannulas were connected via polyethylene tubing (Plastics One) to a subcutaneously implanted osmotic minipump (Alzet 2002) preloaded with the treatment agent. Vehicle (sterile saline) or XENP345 $\left(0.03 \mathrm{mg} \cdot \mathrm{kg}^{-1} \cdot \mathrm{d}^{-1}\right.$, representing a $5: 1$ ratio of XENP345:LPS) was preloaded along with LPS onto pump and infused for 2 weeks ( $n=6$ per group).

Rotational behavior analyses. At 1,2, and 3 weeks after 6-OHDA lesion, amphetamine-induced rotational behavior was monitored in a glass cylinder (diameter, $24.5 \mathrm{~cm}$ ). Animals received $2.5 \mathrm{mg} / \mathrm{kg} \mathrm{D}$-amphetamine (Sigma) intraperitoneally, and $60 \mathrm{~min}$ after the injection, rotational asymmetry was monitored for $20 \mathrm{~min}$. Rotation toward the lesion (ipsilateral) was scored as positive and net rotational asymmetry score was expressed as full body turns per minute.

Perfusion and tissue processing for histology. At 3 weeks after 6-OHDA lesion or 8 weeks after start of LPS infusion, animals were deeply anesthetized with pentobarbital and intracardially perfused with $300 \mathrm{ml}$ of heparinized PBS, pH 7.4, followed by $500 \mathrm{ml}$ of $4 \%$ paraformaldehyde in $\mathrm{PBS}, \mathrm{pH}$ 7.4. Brains were postfixed for $24 \mathrm{~h}$ in the same paraformaldehyde solution and cryoprotected in 20\% sucrose in PBS for 18-24 h. Coronal sections ( $40 \mu \mathrm{m}$ thickness) were cut through the striatum and substantia nigra pars compacta on a Leica (Nussloch, Germany) cryostat and mounted on glass slides (SuperFrost Plus; Fisher Scientific, Houston, TX) for immunohistological analyses and stereological estimate of DA neuron number in a fixed $200 \mu \mathrm{m}$ area in SNpc.

Immunohistochemistry of brain sections. Sections on glass slides were fixed for an additional $15 \mathrm{~min}$ in $4 \%$ paraformaldehyde, followed by a PBS rinse, $\mathrm{pH}$ 7.4. Mounted sections (rather than the standard freefloating sections) were chosen because of the critical importance of maintaining right/left hemisphere orientation at all times for comparison of ipsilateral (lesioned) to contralateral (unlesioned) side in both the unilateral 6-OHDA and LPS lesion models. Even with the use of standard brain notching techniques, reestablishing correct right/left orientation before mounting free-floating sections would have been technically laborious and prone to error. Before immunohistochemistry, sections were incubated in $0.2 \mathrm{M}$ glycine, $\mathrm{pH} 7.4$, for $30 \mathrm{~min}$ to minimize tissue autofluorescence caused by the aldehyde fixative. Pilot experiments were conducted using $40 \mu \mathrm{m}$ brain sections from control (unlesioned rats) to establish the optimum blocking and antibody incubation times to completely penetrate the section all the way to the $2 \mu \mathrm{m}$ bottom guard zone used in the stereological analyses of neuron number. Sections were permeabilized for $35 \mathrm{~min}$ in TBS containing $0.3 \%$ Triton X-100 and $1 \%$ normal goat serum (NGS), followed by blocking for $60 \mathrm{~min}$ in TBS containing $1 \%$ NGS. Primary antibody incubations were done for $48 \mathrm{~h}$ at $4^{\circ} \mathrm{C}$ in TBS containing $0.1 \%$ Triton X-100 and $1 \%$ NGS. Secondary antibody incubations were performed for $4 \mathrm{~h}$ at room temperature in the same dilution buffer. Nuclei were counterstained with $0.5 \mu \mathrm{g} / \mathrm{ml}$ Hoechst 33258. Sections were coverslipped with aqueous-based mounting medium with antifade reagent (Biomeda, Foster City, CA).

Nigral DA neuron counts. StereoInvestigator analyses software (MicroBrightField, Williston, VT) was used to perform unbiased stereological counts of NeuN/TH-immunoreactive (NeuN/TH-IR) cell bodies in the SNpc using the optical fractionator method (West et al., 1991) for both LPS and 6-OHDA studies. The boundary of SNpc was defined according to previous anatomical demarcation in the rat (German and Manaye, 1993). For analysis, the treatment of the various brain sections was blinded to the observer. Cells were counted with a $100 \times$ oil immersion objective (1.3 numerical aperture) using an Olympus (Tokyo, Japan) BX61 microscope. Random and systematic counting frames (each $50 \times$ 
$50 \times 5 \mu \mathrm{m}$ with $2 \mu \mathrm{m}$ upper and lower guard zones) on cryosections (40 $\mu \mathrm{m}$ serial sections placed four per slide) obtained from over 40 rats through the extent of $\mathrm{SNpc}$ (from $\mathrm{AP},-3.3$ to $-5.3 \mathrm{~mm}$ behind bregma) were sampled using a $20 \mu \mathrm{m}$ optical dissector. We systematically chose to stain every other slide for $\mathrm{TH} / \mathrm{NeuN}$ and the intervening slide for complement 3 receptor (OX-42 antibody). A dopaminergic neuron was defined as a NeuN/TH-immunoreactive cell body with a clearly visible unstained nucleus. For estimating total neuron number (number of NeuN-positive cell), a cell was defined as a soma with a nucleolus in focus within the counting frame. A microglial cell was defined as an OX-42immunoreactive cell with processes (ramified) or without processes (ameboid shape).

Striatal TH fiber density. Fluorescence intensity of THimmunoreactive fibers above a fixed threshold using entorhinal cortex for background subtraction was used to estimate striatal TH-fiber density. For this analysis, cryosections were taken from a region $1 \mathrm{~mm}$ anterior to olfactory bulb, through the caudate-putamen $(\mathrm{CPu})$ complex, and ending $1 \mathrm{~mm}$ posterior to $\mathrm{SNpc}$. Areas at the striatal lesion site (AP, $-1.2 \mathrm{~mm}$ from bregma; ML, $-3.9 \mathrm{~mm}$ from midline in right hemisphere; and DV, $-5 \mathrm{~mm}$ below surface of dura) (Paxinos et al., 1985) devoid of cellular material were excluded from the analysis and normalized for comparison to the contralateral (unlesioned side) according to standard practice.

Mesencephalic mixed neuron/glia cultures. Primary rat ventral mesencephalic neuron/glia cultures were prepared by modification of a published protocol (Liu et al., 2000). Briefly, ventral mesencephalic tissues were dissected from embryonic day 14 (E14) Fischer 344 rats and dissociated with mild mechanical trituration. Cells were plated into four-well chamber slides (two $25 \mu \mathrm{l}$ microislands per well at a density of $7.5 \times 10^{5}$ cells $/ \mathrm{ml}$ ) according to the method of Takeshima et al. (1994) or in 96-well culture plates $\left(100 \mu \mathrm{l} /\right.$ well at a density of $2 \times 10^{5}$ cells $\left./ \mathrm{ml}\right)$ precoated with poly-D-lysine $(0.1 \mathrm{mg} / \mathrm{ml})$ and laminin $(20 \mu \mathrm{g} / \mathrm{ml})$ in DMEM/F12 supplemented with $10 \%$ fetal bovine serum (FBS), $1 \mathrm{~g} / \mathrm{L}$ glucose, $2 \mathrm{~mm}$ L-glutamine, $1 \mathrm{~mm}$ sodium pyruvate, $100 \mu \mathrm{M}$ nonessential amino acids, $50 \mathrm{U} / \mathrm{ml}$ penicillin, $50 \mu \mathrm{g} / \mathrm{ml}$ streptomycin, and $10 \mathrm{ng} / \mathrm{ml}$ basic fibroblast growth factor (bFGF). Cultures were maintained at $37^{\circ} \mathrm{C}$ in a humidified atmosphere of $5 \% \mathrm{CO}_{2} / 95 \%$ air. Cultures were replenished $2 \mathrm{~d}$ later with $0.5 \mathrm{ml} /$ well (chamber slides) or $0.1 \mathrm{ml} /$ well (96-well plate) fresh medium lacking bFGF and were used for treatment $5 \mathrm{~d}$ later. For treatment (in duplicate or triplicate) with LPS or 6-OHDA, the cultures were maintained in $0.5 \mathrm{ml} /$ well (chamber slides) or $0.1 \mathrm{ml} /$ well (96-well plate) of medium supplemented with $2.5 \%$ FBS and lacking bFGF.

Quantitative TNF ELISA. The culture medium was collected after treatment of cells with LPS or 6-OHDA as indicated. The quantities of TNF- $\alpha$ were measured with a mouse TNF- $\alpha$ ELISA kit from Biosource/ Invitrogen per the manufacturer's instructions.

Immunocytochemistry in EVM cultures. Neurons were stained with anti-MAP2b antibody to detect both somata and neurites or anti-NeuN antibody to detect somata only. Dopaminergic neurons were detected with anti-TH antibody. GABAergic neurons were identified with antiGABA antibody. Microglia were detected with an anti-complement type 3 receptor antibody (OX-42), CD-45 antibody, or FITC-BS-1 isolectin B4. Cells were fixed with $4 \%$ paraformaldehyde, permeabilized with TBS containing 3\% gelatin from cold water fish skin (Sigma), 1\% BSA, and $0.3 \%$ Triton X-100, blocked with TBS containing 3\% gelatin from cold water fish skin and $1 \%$ BSA. Primary antibody incubations were done for $2 \mathrm{~h}$ at room temperature with primary antibodies diluted in TBS containing antibody diluent (TBS containing 3\% gelatin from cold water fish skin, $1 \% \mathrm{BSA}$, and $0.1 \%$ Triton X-100), anti-MAP2b (1:400), antiNeu-N (1:400), anti-TH (1:250), OX-42 (1:60), anti-CD-45 (1:600), anti-GABA (1:1500), FITC-BS-1 isolectin B4 $(0.1 \mathrm{mg} / \mathrm{ml})$, or anti-GFAP (1:1000). Except for FITC-isolectin B4, the bound primary antibody was visualized on an Olympus BX61 fluorescence microscope after incubation with an appropriate Invitrogen Alexa-conjugated secondary antibody (1:1000). Images were captured with either a CoolSnap CCD ES monochromatic or CF color camera and analyzed with MetaMorph software (Universal Imaging Systems, West Chester, PA). For analysis, the treatment of the various cultures was blinded to the observer. Counts were performed in a minimum of six fields per well per treatment condition. Each experimental set was repeated two to three times.

Uptake assays for tritiated DA or GABA. Neurotransmitter uptake was measured using published protocols (Gao et al., 2002). Mixed neuron/ glia cultures were washed two times with $100 \mu$ l of Krebs-Ringer buffer (containing $16 \mathrm{~mm}$ sodium phosphate, $119 \mathrm{~mm}$ sodium chloride, $4.7 \mathrm{~mm}$ potassium chloride, $1.8 \mathrm{~mm}$ calcium chloride, $1.2 \mathrm{~mm}$ magnesium sulfate, $1.3 \mathrm{~mm}$ EDTA, and $5.6 \mathrm{~mm}$ glucose; $\mathrm{pH}$ 7.4). For DA and GABA uptake, the cultures were incubated for $15 \mathrm{~min}$ at $37^{\circ} \mathrm{C}$ with $50 \mu \mathrm{l}$ of $10 \mu \mathrm{M}$ $\left[{ }^{3} \mathrm{H}\right] \mathrm{DA}(30 \mathrm{Ci} / \mathrm{mmol})$ and $50 \mu \mathrm{M}\left[{ }^{3} \mathrm{H}\right] \mathrm{GABA}(90 \mathrm{Ci} / \mathrm{mmol})$ in KrebsRinger buffer, respectively. After being washed three times with $100 \mu \mathrm{l}$ of ice-cold Krebs-Ringer buffer, the cells were collected in $50 \mu \mathrm{l}$ of $1 \mathrm{~N}$ sodium hydroxide, combined with $1 \mathrm{ml}$ of scintillation fluid, and radioactivity was counted with a liquid scintillation counter. Nonspecific uptake was determined in parallel wells that received both the tritiated tracer and $10 \mu \mathrm{M}$ mazindol (to block specific DA uptake) or $2 \mathrm{~mm}$ $\beta$-alanine (to block glial GABA uptake) (Mabjeesh et al., 1992). Each treatment condition was done in triplicate. Each experimental set was repeated at least two times.

Statistical analyses. Differences among means were analyzed using one-way ANOVA. When ANOVA showed significant differences, comparisons between means were tested by the Tukey-Kramer multiplecomparison post hoc test. Left versus right differences from the same animals were analyzed using two-tailed paired Student's $t$ test. Values expressed are the group mean \pm SEM. For culture experiments, differences in TH cell number among the different groups were analyzed by ANOVA followed by the Newman-Keuls post hoc test for $p$ values significance. Values expressed are the mean $\pm \mathrm{SD} ;{ }^{\star} p<0.05 ;{ }^{*} p<0.01$; ${ }^{* * *} p<0.001$.

\section{Results}

\section{Blocking soluble TNF signaling in vivo provides neuroprotection to dopamine neurons from 6-OHDA- induced death and attenuates rotational behavior}

TNF levels have been reported to increase in striatum and substantia nigra of 6-OHDA-injected rats (Mogi et al., 1999). To determine whether TNF signaling has a critical role in nigral DA neuron loss in vivo, we tested the ability of a DN-TNF inhibitor (XENP345), to provide neuroprotection from 6-OHDA-induced lesions in rats. 6-OHDA neurotoxin rodent models of PD are characterized by increased oxidative stress and striatal terminal die-back followed by loss of DA cell bodies within the substantia nigra (Przedborski, 2005; Przedborski and Ischiropoulos, 2005). In these experiments, a lesion was induced by a unilateral intrastriatal preterminal injection of 6-OHDA into rats (Kirik et al., 1998). Immediately after administration of 6-OHDA (or vehicle) into the striatum, a cannula connected to an osmotic minipump preloaded with XENP345 or saline was stereotaxically positioned into the ipsilateral striatum or ipsilateral SNpc. Using unbiased design-based stereology, the number of TH/MAP2b-positive soma was estimated within the substantia nigra 3 weeks after administration of the 6-OHDA striatal injection. We found that administration of the XENP345 into the nigra rescued $\sim 50 \%$ of the nigral DA neurons from 6-OHDA-induced death and prevented the decrease in $\mathrm{TH}$ fiber density (Fig. $1 a-e$ ). In contrast, no statistically significant difference was detected between groups when rats were implanted with striatal pumps preloaded with vehicle or XENP345. These findings suggest that the key TNF signaling events mediating neurodegeneration of DA neurons occur at the cell somata in the region of the substantia nigra pars compacta.

As a physiological measure of 6-OHDA-induced striatal DA depletion, we tested amphetamine-induced rotational behavior weekly for 3 weeks after the 6-OHDA lesion. We found that the protection of DA neurons in SNpc achieved with nigral delivery of XENP345 correlated with attenuated ipsiversive circling be- 


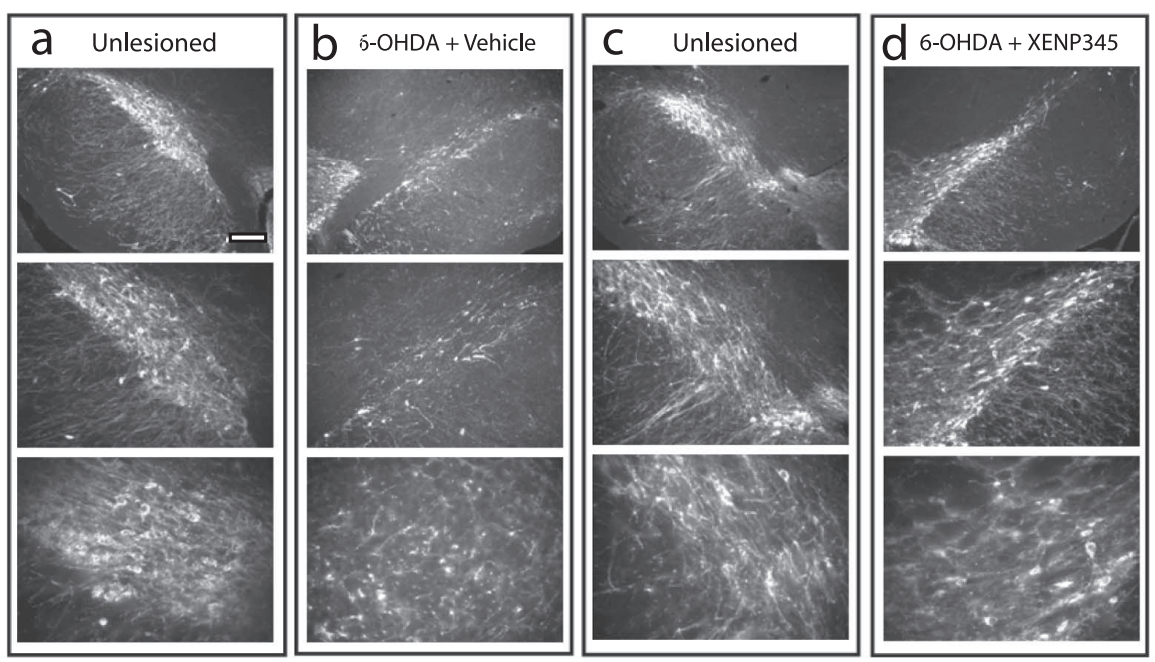

*

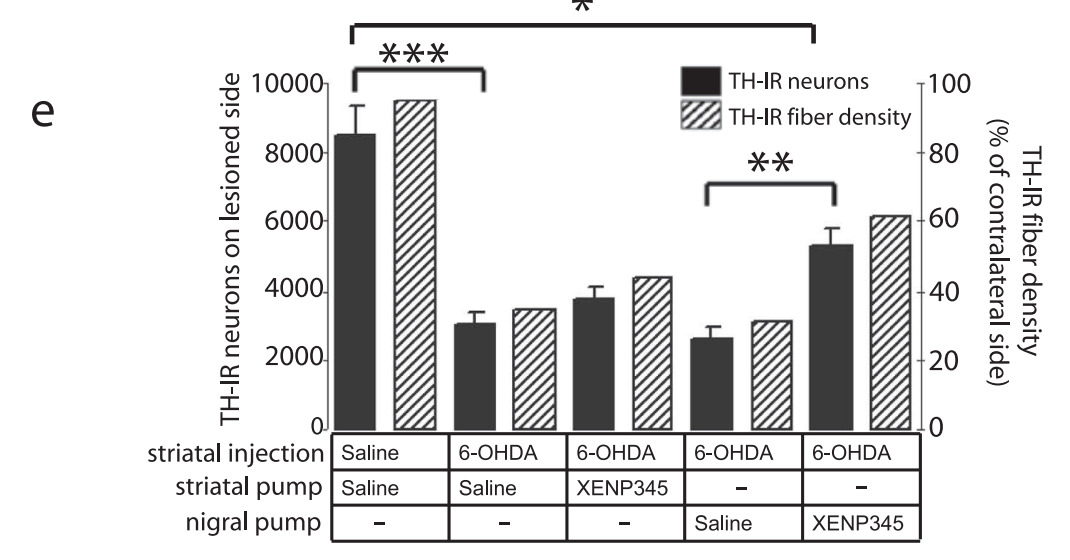

$f$

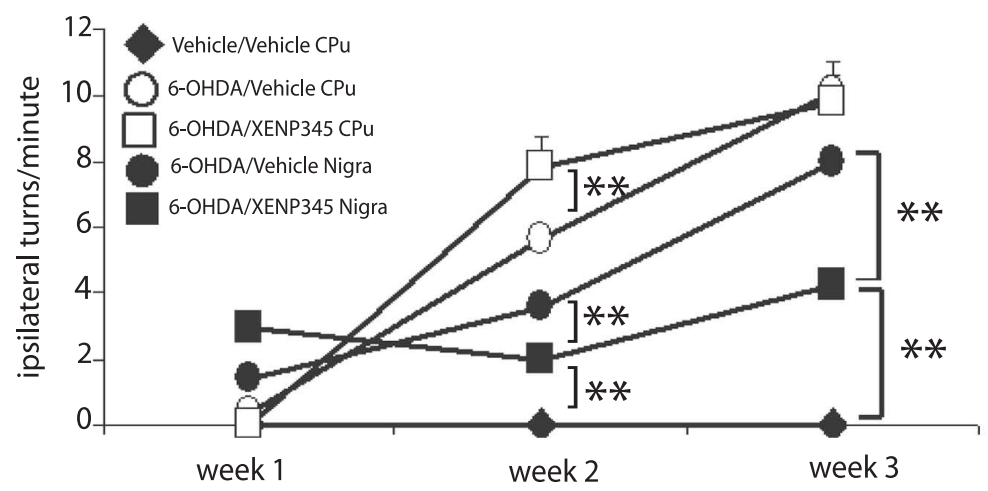

Figure 1. Blocking TNF signaling in the nigra attenuates striatal 6-OHDA-induced loss of nigral DA neurons and drug-induced rotational behavior. A unilateral striatal lesion was induced by injecting 6-OHDA (20 $\mu \mathrm{g})$ into the CPu complex of young adult rats; mock-lesioned animals received an injection of saline. Animals were stereotaxically implanted with an ipsilateral striatal or nigral indwelling cannula connected to an Alzet 2002 osmotic pump to deliver saline vehicle or XENP345 $\left(0.08 \mathrm{mg} \cdot \mathrm{kg}^{-1} \cdot \mathrm{d}^{-1}\right)$ over a 2 week period. Animals were anesthetized, and brains were fixed for immunohistochemical analyses of TH/NeuN-IR neurons by intracardiac perfusion 3 weeks after the lesion. Panels from top to bottom represent increasing magnification of representative brain sections used to obtain stereological estimates of nigral DA neuron number in 6-OHDA-lesioned animals implanted with pump preloaded with saline [contralateral unlesioned side $(\boldsymbol{a})$; lesioned/pump side $(\boldsymbol{b})$ ] or implanted with nigral pump preloaded with XENP345 [contralateral unlesioned side (c); lesioned/pump side (d)]. Scale bar: top panel, $100 \mu \mathrm{m}$; middle panel, $50 \mu \mathrm{m}$; bottom panel, $10 \mu \mathrm{m} . \boldsymbol{e}$, Stereological estimate of DA neuron number (TH/NeuN-IR cells) in SNpcexpressed as a percentage of the contralateral side (solid bars). Statistical significance was evaluated by ANOVA followed by post hoc comparison test between groups and to unlesioned control group. Values expressed are group mean + SEM; ${ }^{*} p<0.05 ;{ }^{* *} p<0.01 ;{ }^{* * *} p<0.001$. Fluorescence intensity of TH-immunoreactive fibers was used to estimate striatal TH-fiber density (hatched bars) on the lesioned side, expressed as a percentage of the unlesioned contralateral side. $f$, As a physiological measure of striatal DA depletion in mockor 6-OHDA-lesioned animals, rotational behavior induced by an intraperitoneal injection of $2.5 \mathrm{mg} / \mathrm{kg}$ amphetamine was measured weekly in all animals and expressed as the number of ipsilateral turns per minute. Values expressed are group mean $\pm \mathrm{SEM}$; ${ }^{* *} p<0.01$. havior induced by D-amphetamine (2.5 $\mathrm{mg} / \mathrm{kg}$, i.p.) (Fig. 1f). These data suggest that TNF may be crucial for the progressive phase of 6-OHDA-induced dopaminergic cell loss, and support a role for TNF signaling in DA neuron loss induced by oxidative neurotoxins.

\section{Blocking soluble TNF signaling in vivo during a chronic neuroinflammatory stimulus protects DA neurons from death} Because inhibition of solTNF signaling was efficacious in protecting DA neurons from death induced by the oxidative neurotoxin 6-OHDA, we hypothesized that a TNF-dependent neuroinflammatory or neurotoxic component is required for robust killing of nigral DA neurons. To test this, we used a purely neuroinflammatory in vivo model of $\mathrm{PD}$ and compared survival of TH+ neurons in SNpc after infusion of LPS alone or LPS and XENP345. Previous studies by Gao et al. (2002) reported that a 2 week supranigral infusion of LPS ( $5 \mathrm{ng} / \mathrm{h}$ ) increased the number of complement 3 receptor (Ox-42 antibody) immunoreactive microglia with rod-like morphology as early as $3 \mathrm{~d}$ after the start of LPS infusion, which subsequently peaked at 2 weeks and persisted for 8 weeks. This microglial activation profile is consistent with this being a chronic neuroinflammatory model. We have confirmed the persistent presence of $\mathrm{Ox}$-42-positive ameboidshaped microglia at week 8 after a 2 week chronic LPS infusion into SNpc (Fig. 2). We reasoned that because TNF is a potent activator of microglia (Aggarwal et al., 2000; Hinkerohe et al., 2005) and a known mediator of LPS action in peripheral tissues (Beutler, 2005), chronic infusion of DN-TNFs to block solTNF signaling during an inflammatory stimulus might prevent TH-positive cell loss in the SNpc of LPS-infused rats. Unbiased stereological measurements of TH-IR/NeuN neuron soma and fluorescence densitometry of TH-positive fiber density revealed that coinfusion of XENP345 (70 ng/h) with LPS ( $5 \mathrm{ng} / \mathrm{h}$ ) for $14 \mathrm{~d}$ into rat substantia nigra rescued $\sim 50 \%$ of the LPS-induced nigrostriatal degeneration measured 8 weeks after the start of infusion (Fig. 2c). In addition, rescue of DA neurons was accompanied by a decreased number of C3R-IR microglia in SNpc (Fig. 2a). Infusion of XENP345 alone, chronically or in a single bolus $(20 \mu \mathrm{g})$, did not induce microgliosis and was not associated with any tissue damage (Fig. 2a) consistent with a lack of TNF-like agonistic activity of DN-TNFs published in previous work by Steed et al. 
(2003). Together with our findings in the 6-OHDA model, these data indicate that solTNF signaling is a primary mediator of DA neuron loss in vivo induced by either neuroinflammatory agents or oxidative neurotoxins, and that inhibition of solTNF can reduce neuroinflammation and subsequent neurodegeneration.

\section{Soluble TNF is a critical mediator of LPS- and 6-OHDA-induced dopaminergic neuron loss in vitro} Given the fact that in vivo administration of TNF inhibitors selective for solTNF signaling rescued only $50 \%$ of DA neurons from LPS- or 6-OHDA-induced degeneration, it was important to determine whether additional rescue of DA neurons could be achieved by also blocking signaling by tmTNF. To determine the contribution from each form of the ligand to the death of DA neurons in LPS-treated embryonic (E14) rat ventral mesencephalon (EVM) cultures, we compared sparing of TH neurons by two inhibitors with different modes of action. We first measured LPS-induced solTNF production in the medium by quantitative ELISA to determine the doses of TNF inhibitors needed to block TNF signaling during an LPS stimulus. We found that treatment of EVM cultures with $10 \mathrm{ng} / \mathrm{ml}$ LPS led to rapid release of solTNF which peaked at $\sim 500 \mathrm{pg} / \mathrm{ml}$ (Fig. 3a). In pilot experiments, we next confirmed the lack of toxic effects of DN-TNFs alone (XENP345 and others) or etanercept alone and determined the optimal dosing of TNF inhibitors necessary to completely block DA neuron death induced by exogenous solTNF (data not shown). Next, we treated EVM neuron/glia cultures with $10 \mathrm{ng} / \mathrm{ml}$ LPS (Fig. $3 b$ ) in the presence of either XENP345 (3 or $200 \mathrm{ng} / \mathrm{ml}$ ) to block solTNF exclusively (Steed et al., 2003) or with the soluble decoy receptor etanercept (Fc-TNFR2; $100 \mathrm{ng} / \mathrm{ml}$ ) to block both solTNF and tmTNF signaling (Scallon et al., 2002; Agnholt et al., 2003; Mitoma et al., 2005). Four days after LPS stimulation, DA neuron survival was measured by counting tyrosine hydroxylase and MAP-2b colabeled neurons (Fig. 3b). We found that blocking TNF signaling with either inhibitor during an LPS stimulus attenuated LPS-induced loss of DA neuron number (Fig. 3c) and their neurites (Fig. $3 b$ ) equally well and by approximately onehalf, indicating that the gradual loss of TH-positive neurons induced by LPS treatment and dependent on TNF signaling is mediated exclusively by solTNF, because blocking of both tmTNF and solTNF with etanercept yielded no further rescue compared with XENP345. The slight differences between XENP345 and etanercept in ability to rescue DA neurons in vitro may be related to pharmacokinetic differences, because the doses of both TNF

a

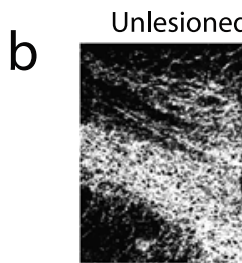

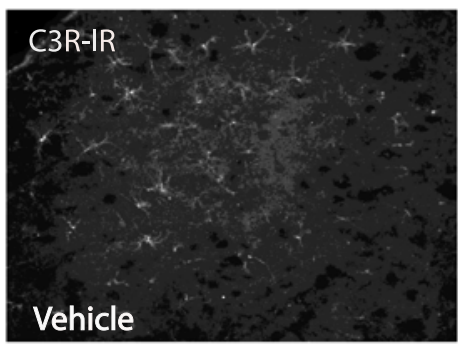
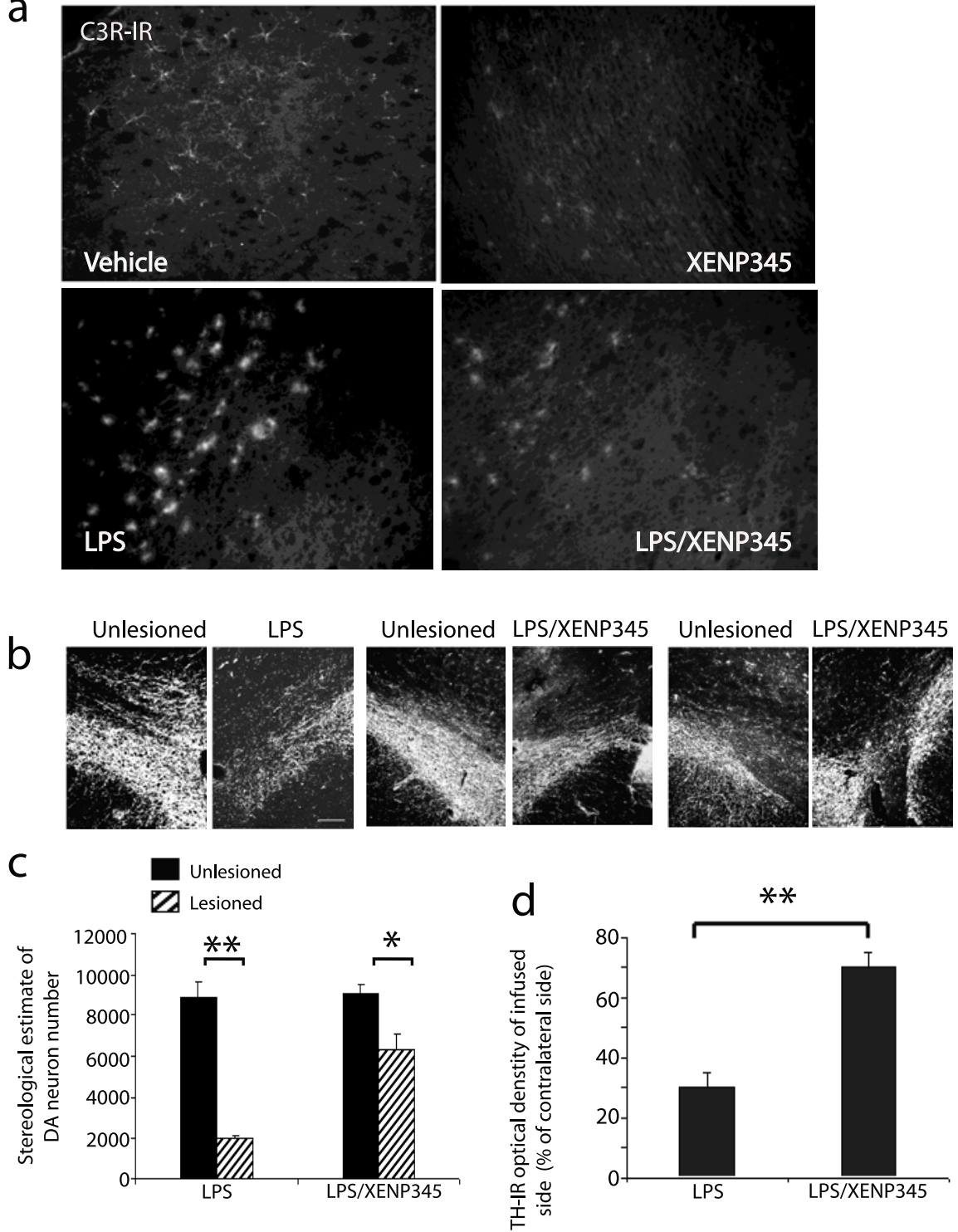

Figure 2. Inhibition of soluble TNF signaling in vivo with XENP345 protects DA neurons from LPS-induced death. Low-dose LPS ( $5 \mathrm{ng} / \mathrm{h}$ in normal saline) was infused chronically for 2 weeks into the substantia nigra of CDF/Fischer 344 rats with or without XENP345 (70 ng/h in normal saline). $\boldsymbol{a}$, Representative sections of microglia activation detected by C3R-positive cells in nigral sections from rat SNpc chronically infused with vehicle, LPS, LPS plus XENP345, or a single bolus of XENP345 (20 $\mu \mathrm{g})$. Scale bar, 10 $\mu \mathrm{m}$. A ramified (resting) morphology is evident in vehicle and XENP345 alone brains. LPS/XENP345 coinfused SNpc displayed fewer ameboid (activated) microglia compared with LPS alone. $\boldsymbol{b}$, Representative sections of TH-IR from an animal infused with LPS and two different animals coinfused with LPS/XENP345. Scale bar, $100 \mu \mathrm{m}$. c, Stereological estimates of nigral DA neuron number (TH/NeuN-coimmunoreactive neurons) after LPS or LPS/XENP345 infusion in SNpc on the unlesioned (contralateral) side in solid bars and lesioned (ipsilateral) side shown in gray hatched bars. Left versus right differences from the same animals were analyzed using two-tailed paired Student's $t$ test. Values were expressed as the group mean \pm SEM; ${ }^{*} p<0.05,{ }^{* *} p<0.01$, significantly different from unlesioned side. $\boldsymbol{d}$, Striatal TH-IR optical density on infused side (expressed as a percentage of contralateral side). Values expressed are the mean $\pm \mathrm{SEM}$; ${ }^{* *} p<0.01$, significantly different from LPS-only infused group.

inhibitors used in vitro were in excess of that needed to neutralize the amount of secreted solTNF measured by ELISA in response to the LPS challenge (Steed et al., 2003). Given that solTNF and tmTNF bind R1 and R2 with different affinities, and the normal biological role of TNF and its receptors in the nigrostriatal pathway is not at all clear, our findings that XENP345 significantly attenuated the death of DA neurons induced by 6-OHDA or LPS in vivo support a direct and neurotoxic role for solTNF as the relevant microglial-derived neuroinflammatory mediator of nigral degeneration. To determine whether the rescued neurons retained function, we measured tritiated dopamine uptake in 


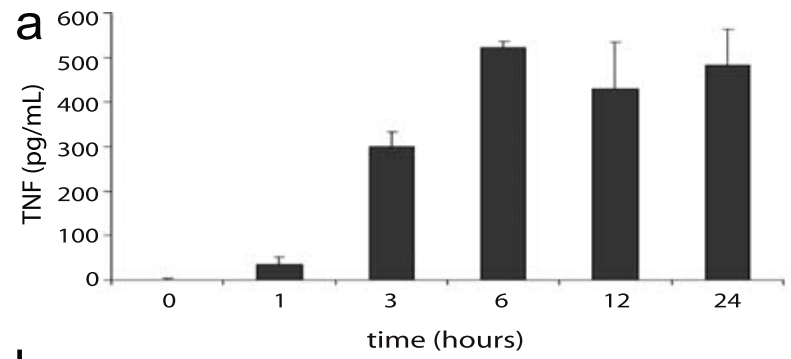

b
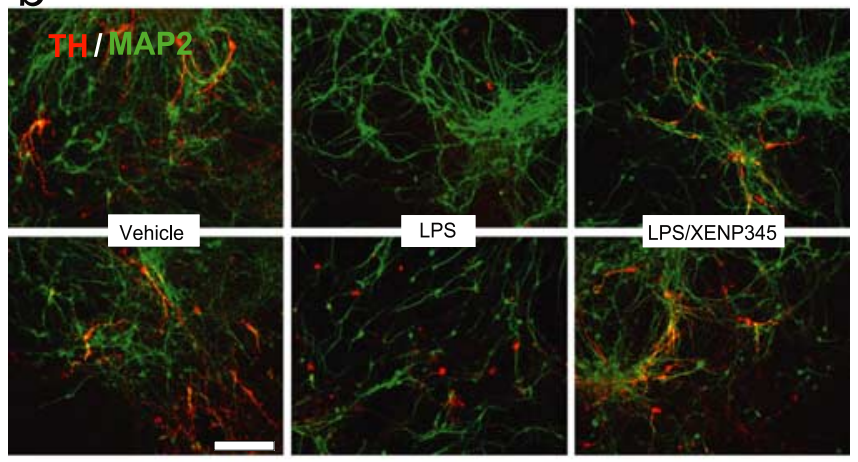

C

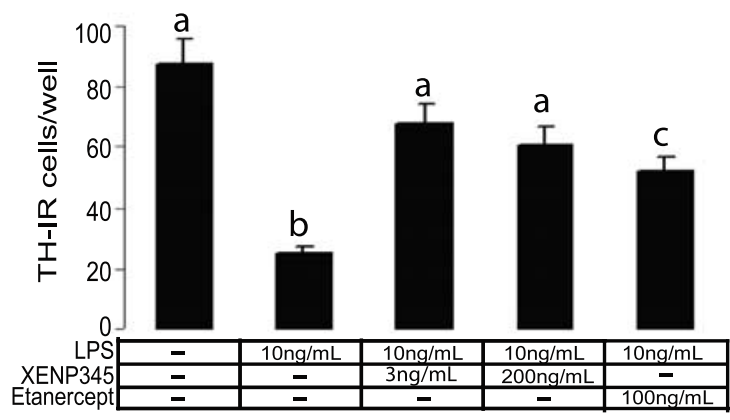

d

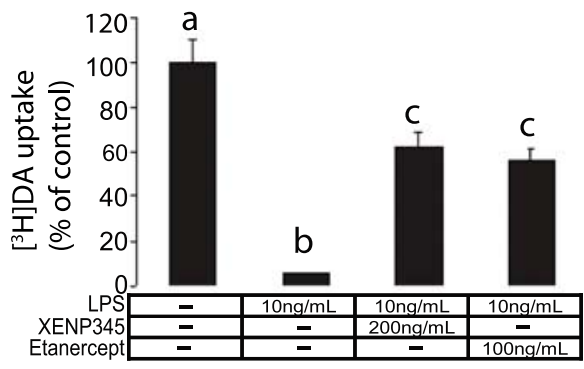

e

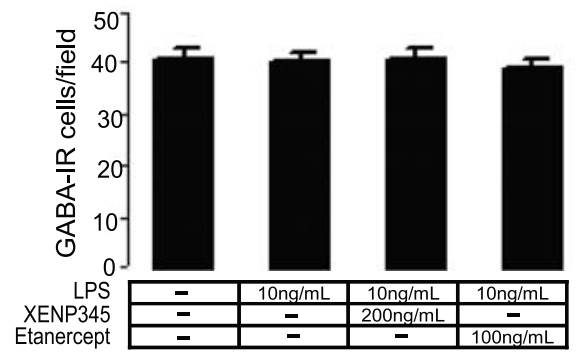

Figure 3. Inhibition of TNF signaling by anti-TNF biologics attenuates LPS-induced neurotoxicity and death of DA neurons in EVM cultures. $\boldsymbol{a}$, Production of solTNF into culture medium elicited by LPS (10 ng/ml) in rat E14 ventral mesencephalon (EVM) neuron/glia cultures was measured by quantitative ELISA. $\boldsymbol{b}$, EVM cultures were treated with LPS $(10 \mathrm{ng} / \mathrm{ml})$ in medium supplemented with $2.5 \%$ FBS (see Materials and Methods) for $4 \mathrm{~d}$ in the presence or absence of solTNF-selective XENP345 or etanercept, a soluble decoy human Fc-TNFR2 receptor that inhibits both tmTNF and solTNF. DA neurons were identified as double-labeled TH/MAP2b cells. Two representative panels are shown for vehicle-, LPS-, or LPS- plus XENP345-treated cultures. Similar results were obtained in a minimum of three independent experiments. c, Quantifica- these cultures. Rescue of TH-positive neurons correlated with increased DA uptake (Fig. 3d). Nonspecific dopamine uptake was measured (and subtracted as background) in these cultures using the specific dopamine transport inhibitor mazindol. In agreement with published observations (Gao et al., 2002), GABAergic neurons were unaffected by LPS treatment (Fig. 3e).

Based on our findings that in vivo inhibition of TNF signaling with XENP345 attenuated 6-OHDA-induced loss of DA neurons and decreased amphetamine-induced ipsiversive circling behavior, we predicted that 6-OHDA-induced neurotoxicity of DA neurons and rescue by XENP345 could also be reproduced in vitro, thus allowing identification of the TNF-dependent mechanisms mediating 6-OHDA-induced neurodegeneration. Therefore, we treated EVM cultures with a concentration range of 6-OHDA (5-100 $\mu \mathrm{M})$ for $24 \mathrm{~h}$ and assayed solTNF release as well as DA uptake in the surviving DA neurons at $4 \mathrm{~d}$ after initial exposure to 6-OHDA in the presence or absence of TNF inhibitors. We found that $20 \mu \mathrm{M} 6-\mathrm{OHDA}$ induced neurotoxicity and death in DA neurons comparable with $10 \mathrm{ng} / \mathrm{ml}$ LPS (Fig. 4) under the same medium conditions and time course of the experiment. Lower concentrations of 6-OHDA did not induce significant DA neuron death (because of the protective effects of $2.5 \% \mathrm{FBS}$ ) and higher concentrations induced excessive death of both DA and GABA neurons (data not shown). As expected, the level of solTNF release $(\sim 50 \mathrm{pg} / \mathrm{ml})$ evoked by this amount of 6-OHDA (Fig. 4a) was lower than that evoked by the inflammogen LPS; however, the robust rescue with XENP345 and etanercept indicated this level of solTNF was sufficient to account for a significant fraction $(>50 \%)$ of the 6-OHDA-induced neurotoxicity (Fig. 4c). Together, these studies are the first to demonstrate a critical role for solTNF signaling in mediating death of DA neurons in vitro and in vivo induced by two different neurotoxins used in rodent models of PD.

TNF-dependent potentiation of microglia activation is not the primary mechanism mediating DA neuron degeneration Given that microglial-derived oxidative stress has been proposed to be involved in the loss of DA neurons in the LPS model (Gao et al., 2002), we investigated the extent to which rescue of DA neurons by inhibition of TNF signaling correlated with attenuated microglia activation (measured using antibodies against the microglial markers complement 3 receptor, isolectin B4, and CD45). EVM cultures were treated with LPS $(10 \mathrm{ng} / \mathrm{ml})$ or with LPS plus XENP345 (200 ng/ml coadded, $100 \mathrm{ng} / \mathrm{ml}$ readded every $24 \mathrm{~h}$ ) to measure microglia activation at $12,24,48$, and $96 \mathrm{~h}$ after addition of LPS. Blocking solTNF signaling with XENP345 did not completely abolish LPS-induced microglia activation, but had its greatest attenuating effects in the early time points $(<24$ h) after LPS stimulation (Fig. 5a,b). In a control experiment, we found that robust induction of microglia activation by $4 \mathrm{ng} / \mathrm{ml}$

$\leftarrow$

tion of DA neuron survival in EVM cultures treated with LPS $(10 \mathrm{ng} / \mathrm{ml})$ for $4 \mathrm{~d}$ in the presence or absence of TNF inhibitors. Similar results were obtained in a minimum of three independent experiments. Values shown are mean \pm SEM; histogram bars with different letters are significantly different $(p<0.05)$. $\boldsymbol{d}$, Uptake of [ $\left.{ }^{3} \mathrm{H}\right]$ dopamine was measured in LPS-treated EVM cultures in the presence or absence of TNF inhibitors. Values shown are background-corrected for nonspecific uptake measured by including mazindol during incubation with the tritiated neurotransmitter. Values shown are mean + SEM; histogram bars with different letters are significantly different $(p<0.05)$. Similar results were obtained in three independent experiments. $\boldsymbol{e}$, GABAergic neuron survival, assessed by counting number of GABA/NeuN doublelabeled cells, was unaffected by LPS treatment. No statistical significant difference was found between groups. Similar results were obtained in two independent experiments. 

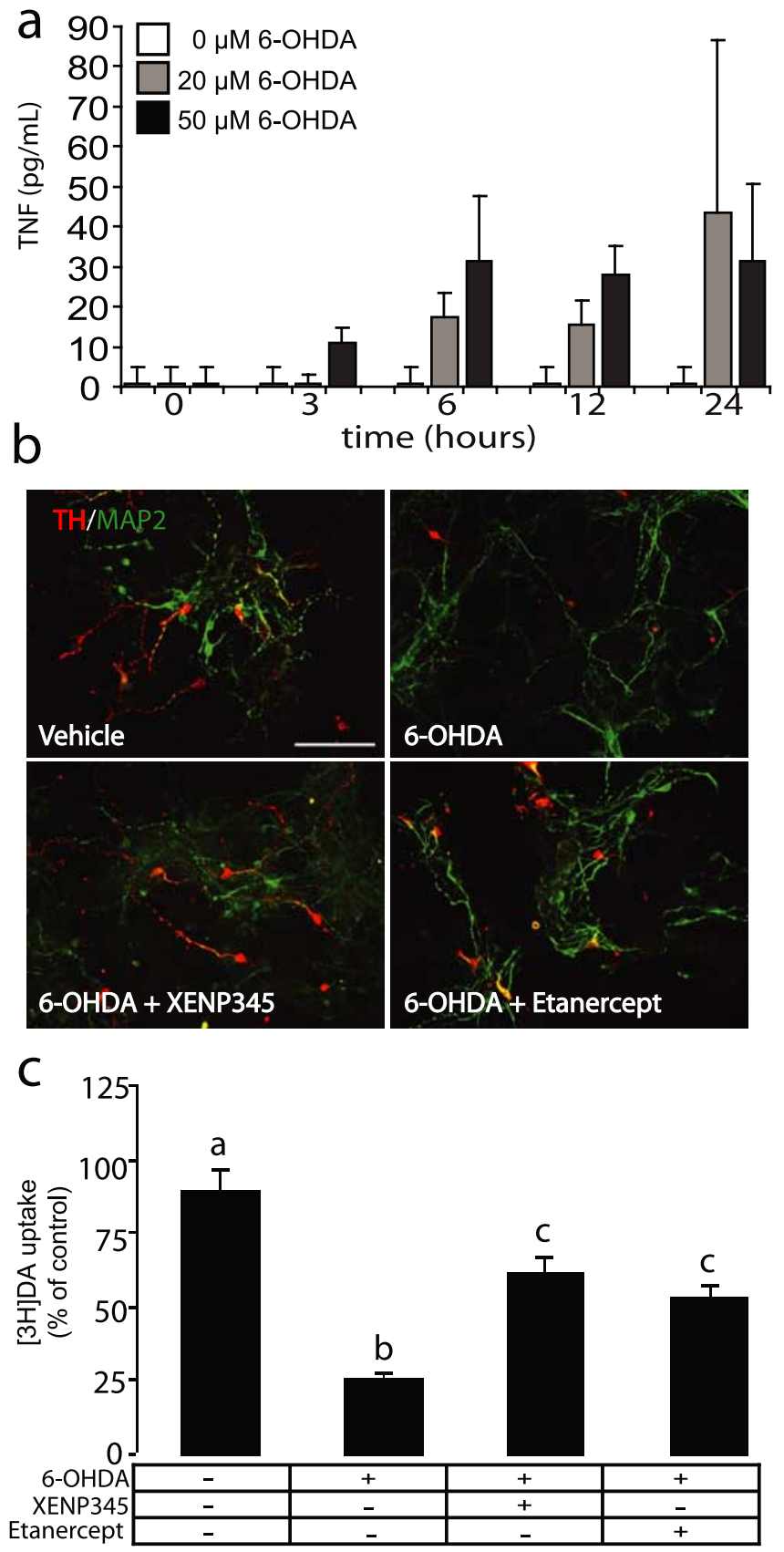

Figure 4. Inhibition of soluble TNF signaling attenuates 6-OHDA-induced death of DA neurons in EVM cultures. $\boldsymbol{a}$, Production of solTNF into culture medium elicited by $6-0 \mathrm{HDA}[0 \mu \mathrm{m}$ (white), $20 \mu \mathrm{m}$ (gray), or $50 \mu \mathrm{m}$ (black)] in ratE14ventral mesencephalon (EVM) neuron/glia cultures was measured by quantitative ELISA. $\boldsymbol{b}$, EVM cultures were treated with 6-hydroxydopamine (20 $\mu \mathrm{M})$ for $24 \mathrm{~h}$ in medium supplemented with $2.5 \% \mathrm{FBS}$ (see Materials and Methods) in the presence or absence of the solTNF-selective XENP345 $(200 \mathrm{ng} / \mathrm{ml})$ or etanercept $(200 \mathrm{ng} / \mathrm{ml})$. DA neuron survival was assessed $96 \mathrm{~h}$ after exposure to 6-OHDA. DA neurons were identified as double-labeled TH/MAP2b cells. Representative panels are shown for vehicle-, 6-OHDA-, 6-OHDA/XENP345-, or 6-0HDA/etanercepttreated cultures. Scale bar, $100 \mu \mathrm{m}$. c, Uptake of $\left[{ }^{3} \mathrm{H}\right]$ dopamine was measured in 6-OHDA-treated EVM cultures in the presence or absence of TNF inhibitors. Values shown are background-corrected for nonspecific uptake measured by including mazindol during incubation with the tritiated neurotransmitter. Values shown are mean \pm SEM; histogram bars with differentletters are significantly different $(p<0.05)$.

solTNF can be inhibited completely by a ratio of XENP345 to solTNF of 50:1 (Fig. 5c), suggesting that partial inhibition of LPSinduced microglia activation with XENP345 was not attributable to incomplete neutralization of solTNF. From these data, we con- a

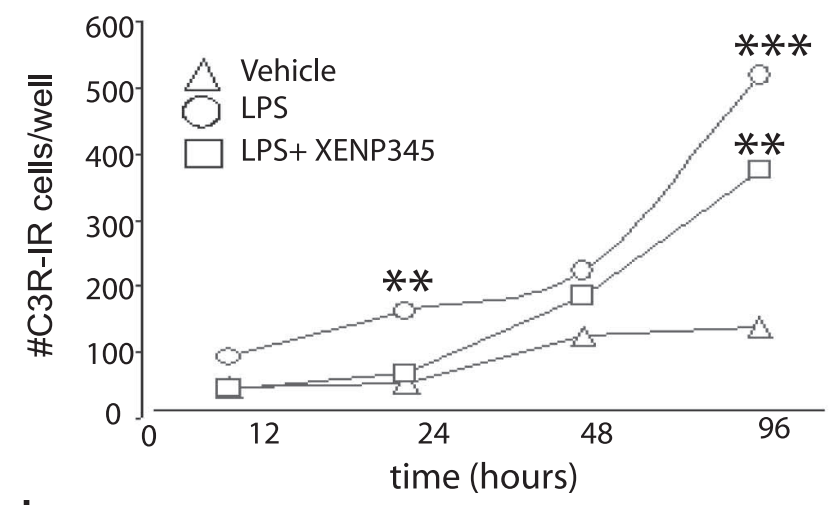

b

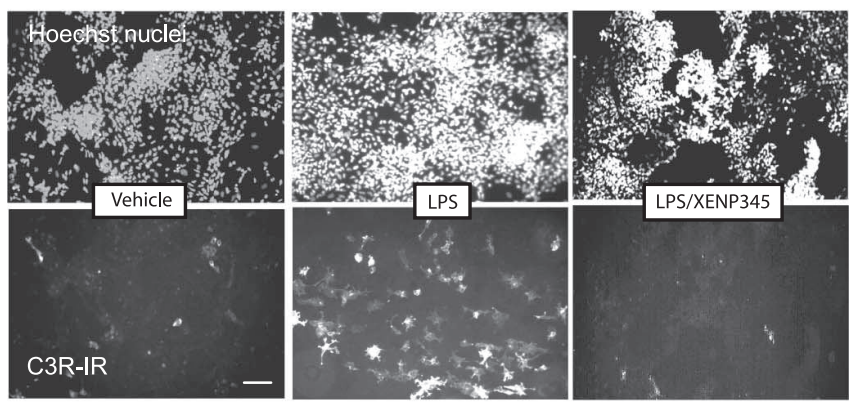

C

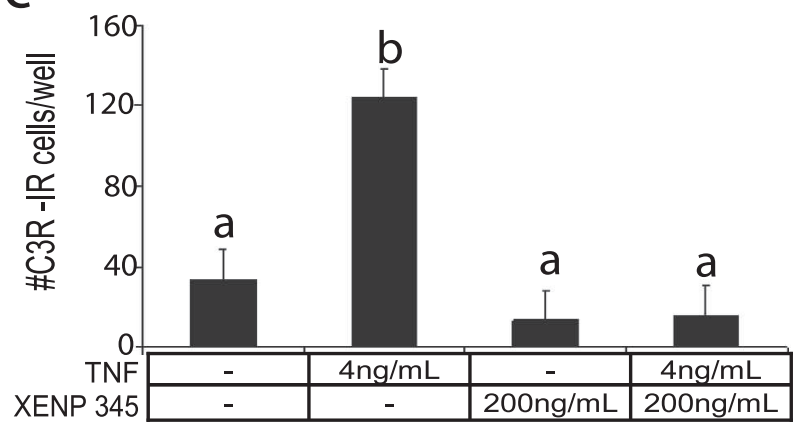

Figure 5. Soluble TNF is a primary mediator of LPS-induced microglia activation and death of DA neurons in ventral mesencephalon neuron/glia cultures. $\boldsymbol{a}$, Quantification of microglia activation (measured by C3R immunoreactivity) induced by LPS treatment for 12,24 , 48 , or $96 \mathrm{~h}$ in the presence (squares) or absence (circles) of XENP345. Vehicle-treated cultures are indicated by triangles. Values are expressed as the mean $\pm \mathrm{SEM}$; error bars are smaller than the symbols; ${ }^{* *} p<0.01,{ }^{* * *} p<0.001$. Similar results were obtained in at least three independent experiments. $\boldsymbol{b}$, Representative images of microglia activation measured after $24 \mathrm{~h}$ of LPS stimulation in the presence or absence of XENP345 (scale bar, $50 \mu \mathrm{m}$ ) is indicated by number of ameboidshaped CR3-immunoreactive microglia in the neuron/glia cell cultures. Similar results were obtained in three independent experiments. $c$, Control experiment showing neutralization of solTNF-induced microglia activation by XENP345 and absence of TNF-like activity when used alone. EVM cultures were treated with TNF $(4 \mathrm{ng} / \mathrm{ml})$ in the presence or absence of XENP345. After $24 \mathrm{~h}$ stimulation, cells were fixed and stained with an antibody specific for C $3 \mathrm{R}$ to quantify the extent of microglia activation. Values shown are mean \pm SEM; histogram bars with different letters are significantly different $(p<0.05)$.

clude that solTNF is required for potentiation of early LPSinduced microglia activation, yet only partially mediates the neuroinflammatory response elicited by LPS. Therefore, we conclude that the critical microglial-derived mediator responsible for DA neuron loss is solTNF, and attenuation of TNF-mediated effects is sufficient to provide significant rescue of DA neurons, because neuroprotection can be achieved with XENP345 despite persistent microglia activation. Experiments to identify the cellular and 
a
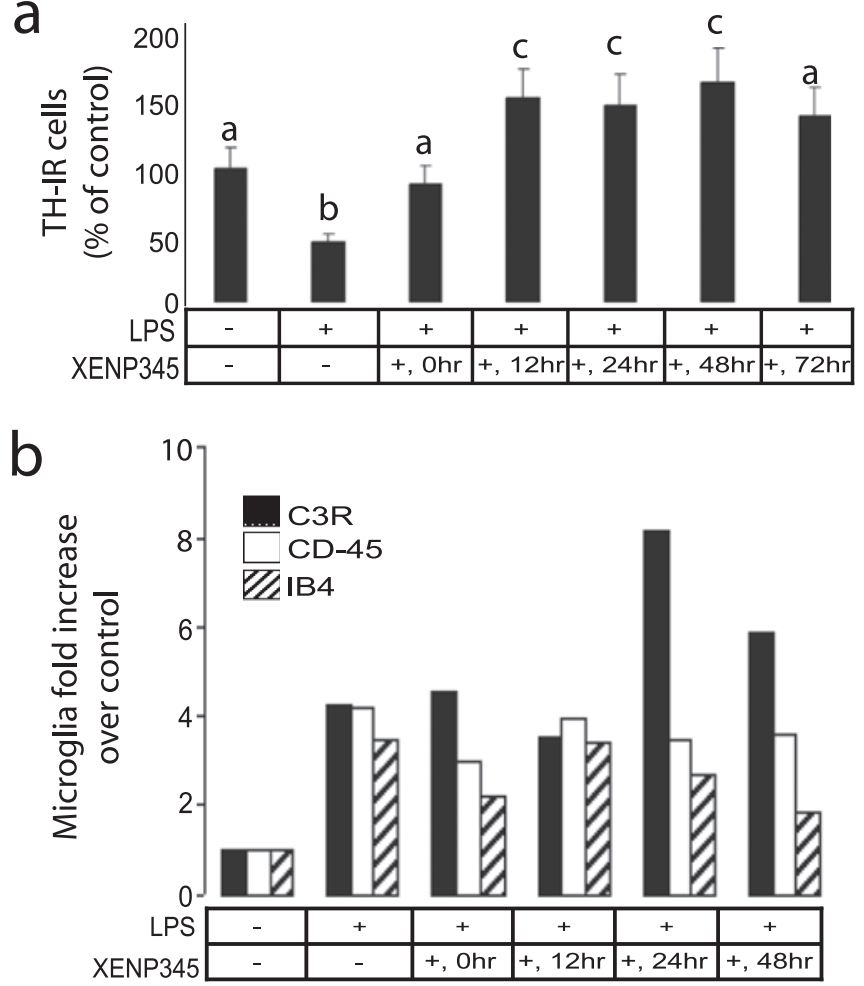

Figure 6. Delayed addition of XENP345 provides robust rescue of DA neurons despite sustained microglia activation. $\boldsymbol{a}$, Quantification of $\mathrm{DA}$ neuron survival after coaddition or delayed addition of XENP345 $(200 \mathrm{ng} / \mathrm{ml})$ after LPS $(10 \mathrm{ng} / \mathrm{ml})$ treatment. Values shown are mean + SEM; histogram bars with different letters are significantly different $(p<0.05)$. Similar results were obtained in two independent experiments. $\boldsymbol{b}$, Quantification of microglia activation in response to LPS $(10 \mathrm{ng} / \mathrm{ml})$ in the presence or absence of XENP345 $(200 \mathrm{ng} / \mathrm{ml})$ using three different immunocytochemical microglial markers (C3R, CD45, IB4). Similar results were obtained in two independent experiments.

signaling mechanisms mediated by TNF and necessary for 6-OHDA-induced neurodegeneration are in progress. It is well known that the action of the oxidative neurotoxin 6-OHDA involves formation of 6-OHDA-paraquinones and leads to GSH (glutathione) depletion of mitochondria (Przedborski, 2005; Przedborski and Ischiropoulos, 2005). Such depletion sensitizes many cells to the toxic actions of TNF via mitochondrial targeting of glycosphingolipids and potentiation of intracellular reactive oxygen species (ROS) accumulation (Garcia-Ruiz et al., 1997; Garcia-Ruiz et al., 2003). Therefore, these TNF-dependent signaling cascades may have important roles in triggering the death of DA neurons and are therefore under investigation.

\section{Delayed inhibition of TNF signaling also rescues DA neurons} from LPS-induced death

We reasoned that because low-dose LPS exposure results in delayed, progressive, and selective death of DA neurons, delayed administration of XENP345 after an LPS insult may still be capable of preventing a substantial portion of DA neuron loss. In these experiments, we treated EVM cultures with LPS, and then added XENP345 after delays of 12-72 h (and readding inhibitor every $24 \mathrm{~h}$ ). Microglial activation and DA neuron survival were measured $4 \mathrm{~d}$ after the initial LPS stimulus. XENP345 was still efficacious in attenuating DA neuron loss (Fig. 6a) even when added several days after the LPS insult. Despite the ability of delayed addition of XENP345 to block DA neuron loss, we found that delayed inhibition of TNF was unable to block sustained LPS- induced microglia activation, as measured by three immunocytochemical markers of microglial activation (Fig. 6b). These data suggest that direct neurotoxicity of microglial-derived TNF on DA neurons, presumably through TNFR1 receptors present on these neurons (McGuire et al., 2001), is likely to be the primary mechanism eliciting delayed death of DA neurons in LPS-treated neuron/glia cultures. However, a second mechanism by which TNF may mediate dopaminergic cell loss is by perpetuating microglial-derived extracellular ROS and reactive nitrogen species (RNS) production, thus increasing oxidative stress on DA neurons and leading to degeneration.

\section{Discussion}

This is the first comprehensive study to employ TNF-selective pharmacological inhibitors in two different models of PD to investigate the direct contribution of the two biologically active forms of TNF in mediating degeneration and death of DA neurons in wild-type rodents. Although it has been known that PD brains possess increased gliosis, cytokine levels, and ROS and RNS, the mechanisms underlying neuroinflammation-mediated DA neuron death have remained unclear. We hypothesized that, independent of the trigger that elicits its production or its cellular source, solTNF might act at two different cellular sites: directly on DA neurons to exert neurotoxic effects and indirectly on microglia to potentiate microglial-derived oxidative stress, and that activation of both routes results in progressive degeneration and loss of DA neurons. Our results that nigral delivery of the soluble TNF inhibitor XENP345 attenuated loss of rat DA neurons, independent of whether this loss is induced by the bacteriotoxin LPS or by the oxidative neurotoxin 6-OHDA, confirm that solTNF is a critical neuroinflammatory mediator involved in the neurodegenerative actions of both LPS and 6-OHDA in vivo. The neuroprotective effects of the immunosuppressant tacrolimus (FK-506) in 6-OHDA-lesioned rats has been ascribed to inhibition of microglial-derived TNF (Mogi et al., 1999), because FK506 has been shown to inhibit secretion of proinflammatory cytokines by macrophages (Keicho et al., 1991). Mechanistically, our results further indicate that TNF drives late signaling events in eliciting neurotoxicity and triggering DA neuron death, because delayed (up to $72 \mathrm{~h}$ ) addition of XENP345 in a $4 \mathrm{~d}$ low-dose LPS treatment regimen can markedly attenuate DA neuron death independent of persistent microglial activation as assessed by several microglial markers. Given that clinical diagnosis of PD in humans occurs only after a significant loss $(>80 \%)$ of nigral DA neuron has occurred, most interventions are in essence "delayed," so these findings have potential clinical relevance; there may be a therapeutic window during which inhibition of TNF signaling could slow disease progression. Consistent with a role of TNF in the pathophysiology of PD, a cohort of early-onset PD patients in Japan have an increased frequency of a polymorphic allele (-1031C) in the TNF gene promoter that results in higher transcriptional activity (Nishimura et al., 2001) and causes them to be high TNF producers. A recent prospective study indicating that regular use of nonsteroidal anti-inflammatory drugs lowers the risk of developing PD by $46 \%$ (Chen et al., 2003) also supports a role for chronic inflammation in triggering or accelerating development of PD in humans.

There are several possible explanations as to why coinfusion of a solTNF inhibitor into the SNpc did not completely abrogate loss of nigral NeuN/TH-positive neurons. We can rule out a role for tmTNF, because etanercept did not yield greater neuroprotection compared with XENP345 in vitro. We can also rule out technical problems with immunohistological detection as a reason for the apparent partial rescue. Specifically, the protocol used 
for staining and detection of NeuN/TH-positive cells throughout the extent of the $40 \mu \mathrm{m}$ sections required for performing stereology using a $20 \mu \mathrm{m}$ optical fractionator was optimized in pilot experiments to ensure complete penetration of primary and secondary antibodies to the bottom guard zone used in the stereology parameters (see Materials and Methods). One possible explanation for the partial rescue that is currently being investigated relates to the potential for limited diffusion of XENP345 away from the delivery site during the chronic infusion. Second, in these experiments, TNF signaling was blocked only in a small region of SNpc for 2 weeks coincident with the chronic LPS infusion or immediately after induction of the intrastriatal 6-OHDA lesion; yet the cascade of neurotoxic and neuroinflammatory events that occurs in these less acute animal models of PD is known to extend beyond the 2-week interval during which TNF signaling was pharmacologically inhibited (Sanchez-Pernaute et al., 2004). Third, partial neuroprotection may be indicative of a component of DA neuron loss that is TNF independent and might involve the action of other proinflammatory cytokines, including interleukin-1 $\beta$ (IL-1 $\beta$ ) or IL-6 (Allan and Rothwell, 2001; Hald and Lotharius, 2005; Nagatsu and Sawada, 2005). To distinguish between these three possibilities, experiments are in progress to determine whether longer or continuous solTNF signaling inhibition across a larger volume of SNpc affords greater neuroprotection to nigral DA neurons, and whether continuous TNF signaling inhibition blocks the progressive phase of 6-OHDA-induced DA neuron death shown to be attenuated by selective COX-2 (cyclooxygenase-2) inhibition (Sanchez-Pernaute et al., 2004). Nonetheless, a reduction of nigral DA neuron death by $50 \%$ with delivery of DN-TNF biologics into the CNS would have a significant and positive impact on delaying progression of DA neuron loss in individuals with PD should these in vivo results in animal models be realized in clinical trials. Because of their size ( $\sim 51 \mathrm{kDa}$ trimers), XENP345 would not be expected to cross the blood-brain barrier, but this possibility is being investigated. Clinically, anti-TNF biologics presently used to treat patients with rheumatoid arthritis (etanercept and infliximab) have been linked to increases in demyelinating disease because of their ability to block tmTNF function (Arnett et al., 2001; Sukal et al., 2006); therefore, a tmTNF-sparing TNF inhibitor for use in PD may be a safer therapy in humans.

To date, evaluation of the role of TNF in mediating DA neuron death in mature animals has only been investigated using acute MPTP intoxication in mice deficient in TNF pathway genes (Rousselet et al., 2002; Sriram et al., 2002; Ferger et al., 2004; Leng et al., 2005), and in wild-type mice treated with thalidomide (Ferger et al., 2004), which inhibits synthesis of TNF and many other genes. However, although these early studies implicated TNF signaling in DA neuron death, these null mice are not ideal models in which to critically address the direct role of TNF in nigral degeneration in adult animals. The brain proteome of TNFR double-knock-out mice indicates significant changes in expression of numerous genes (Pejovic et al., 2004), including the redox sensor DJ-1, which itself has been shown to be important in protecting DA neurons from oxidative stress (Goldberg et al., 2005; Kim et al., 2005). Moreover, mice that develop without any TNF signaling display arrested dendritic cell development and blunted systemic inflammatory responses (Pasparakis et al., 1996; Ritter et al., 2003; Sriram et al., 2006). Our own unpublished observations are that treatment of neuron/glia cultures from TNF knock-out (KO) mice with TNF or LPS elicits blunted microglial activation and attenuated DA neuron death compared with treatment of cultures from WT mice (our manuscript in preparation). Therefore, it is impossible to discern whether resistance (or lack thereof) to MPTP injury in adult mice that developed without TNF signaling is a direct result of no TNF production (i.e., in TNF KO) or inability to bind TNF (i.e., in TNFR1R2 double knock-out) and/or from modifications in the function of downstream TNF-dependent targets (including microglia). In summary, although studies with genetic models suggested that pharmacological manipulation of the TNF pathway may offer neuroprotection, our study is the first to directly demonstrate the feasibility and efficacy of this approach; plus the translational value of studies in adult rats with the soluble TNF-selective pharmacological inhibitor XENP345 makes our direct approach biologically and clinically relevant to humans.

Our data demonstrate that solTNF significantly contributes to toxicity and degeneration of DA neurons, independent of the trigger that elicits its production. This raises the exciting possibility that anti-TNF therapy specifically targeted against solTNF may be an effective treatment for prevention or attenuation of PD progression without interfering with important tmTNF functions such as maintenance of immune function and resistance to infection (Olleros et al., 2005). Lastly, we posit that our findings regarding a critical role of TNF signaling in death of DA neurons may be applicable to other neurodegenerative diseases in which the role of neuroinflammation is being intensely investigated both as a contributing factor, and as the basis for development of new vaccination therapies. For example, levels of TNF were found to be elevated in entorhinal cortex coincident with the earliest appearance of pathology (Janelsins et al., 2005) in a triple transgenic Alzheimer's disease (AD) mouse model (3xTg-AD) harboring mutations in presenilin 1, amyloid precursor protein, and tau (Oddo et al., 2003), and chronic exposure to systemic LPS accelerated development of amyloid and tau pathology in these mice (Kitazawa et al., 2005). Given its role as a major effector of LPS action, we hypothesize that TNF is a key mediator of LPS-enhanced neuropathology in these mice, perhaps by promoting mitochondrial dysfunction and activation of apoptotic death cascades. Investigations of TNF-dependent neuroinflammatory mechanisms that exacerbate neuropathology and hasten neuron loss may unveil opportunities for development of new anti-inflammatory therapeutics to treat human neurodegenerative diseases like PD and AD.

\section{References}

Aggarwal BB, Samanta A, Feldmann M (2000) TNF $\alpha$. In: Cytokine reference (Oppenheim JJ, Feldmann M, eds), pp 414-434. London: Academic.

Agnholt J, Dahlerup JF, Kaltoft K (2003) The effect of etanercept and infliximab on the production of tumour necrosis factor alpha, interferongamma and GM-CSF in in vivo activated intestinal $\mathrm{T}$ lymphocyte cultures. Cytokine 23:76-85.

Allan SM, Rothwell NJ (2001) Cytokines and acute neurodegeneration. Nat Rev Neurosci 2:734-744.

Aloe L, Fiore M (1997) TNF-alpha expressed in the brain of transgenic mice lowers central tyroxine hydroxylase immunoreactivity and alters grooming behavior. Neurosci Lett 238:65-68.

Arnett HA, Mason J, Marino M, Suzuki K, Matsushima GK, Ting JP (2001) TNF alpha promotes proliferation of oligodendrocyte progenitors and remyelination. Nat Neurosci 4:1116-1122.

Barcia C, de Pablos V, Bautista-Hernandez V, Sanchez-Bahillo A, Bernal I, Fernandez-Villalba E, Martin J, Banon R, Fernandez-Barreiro A, Herrero MT (2005) Increased plasma levels of TNF-alpha but not of IL1-beta in MPTP-treated monkeys one year after the MPTP administration. Parkinsonism Relat Disord 11:435-439.

Beutler B (2005) The Toll-like receptors: analysis by forward genetic methods. Immunogenetics 57:385-392.

Boka G, Anglade P, Wallach D, Javoy-Agid F, Agid Y, Hirsch EC (1994) 
Immunocytochemical analysis of tumor necrosis factor and its receptors in Parkinson's disease. Neurosci Lett 172:151-154.

Carvey PM, Chen EY, Lipton JW, Tong CW, Chang QA, Ling ZD (2005) Intra-parenchymal injection of tumor necrosis factor-alpha and interleukin 1-beta produces dopamine neuron loss in the rat. J Neural Transm 112:601-612.

Chen H, Zhang SM, Hernan MA, Schwarzschild MA, Willett WC, Colditz GA, Speizer FE, Ascherio A (2003) Nonsteroidal anti-inflammatory drugs and the risk of Parkinson disease. Arch Neurol 60:1059-1064.

Ferger B, Leng A, Mura A, Hengerer B, Feldon J (2004) Genetic ablation of tumor necrosis factor-alpha (TNF-alpha) and pharmacological inhibition of TNF-synthesis attenuates MPTP toxicity in mouse striatum. J Neurochem 89:822-833.

Gao HM, Hong JS, Zhang W, Liu B (2002) Distinct role for microglia in rotenone-induced degeneration of dopaminergic neurons. J Neurosci 22:782-790.

Garcia-Ruiz C, Colell A, Mari M, Morales A, Fernandez-Checa JC (1997) Direct effect of ceramide on the mitochondrial electron transport chain leads to generation of reactive oxygen species. Role of mitochondrial glutathione. J Biol Chem 272:11369-11377.

Garcia-Ruiz C, Colell A, Mari M, Morales A, Calvo M, Enrich C, FernandezCheca JC (2003) Defective TNF-alpha-mediated hepatocellular apoptosis and liver damage in acidic sphingomyelinase knockout mice. J Clin Invest 111:197-208.

Gayle DA, Ling Z, Tong C, Landers T, Lipton JW, Carvey PM (2002) Lipopolysaccharide (LPS)-induced dopamine cell loss in culture: roles of tumor necrosis factor-alpha, interleukin-1beta, and nitric oxide. Brain Res Dev Brain Res 133:27-35.

German DC, Manaye KF (1993) Midbrain dopaminergic neurons (nuclei A8, A9, and A10): three-dimensional reconstruction in the rat. J Comp Neurol 331:297-309.

Goldberg MS, Pisani A, Haburcak M, Vortherms TA, Kitada T, Costa C, Tong Y, Martella G, Tscherter A, Martins A, Bernardi G, Roth BL, Pothos EN, Calabresi P, Shen J (2005) Nigrostriatal dopaminergic deficits and hypokinesia caused by inactivation of the familial Parkinsonism-linked gene DJ-1. Neuron 45:489-496.

Hald A, Lotharius J (2005) Oxidative stress and inflammation in Parkinson's disease: is there a causal link? Exp Neurol 193:279-290.

Heldmann U, Thored P, Claasen JH, Arvidsson A, Kokaia Z, Lindvall O (2005) TNF-alpha antibody infusion impairs survival of strokegenerated neuroblasts in adult rat brain. Exp Neurol 196:204-208.

Hinkerohe D, Smikalla D, Haghikia A, Heupel K, Haase CG, Dermietzel R, Faustmann PM (2005) Effects of cytokines on microglial phenotypes and astroglial coupling in an inflammatory coculture model. Glia 52:85-97.

Hirsch EC, Hunot S, Hartmann A (2005) Neuroinflammatory processes in Parkinson's disease. Parkinsonism Relat Disord 11 [Suppl 1]:S9-S15.

Hunot S, Dugas N, Faucheux B, Hartmann A, Tardieu M, Debre P, Agid Y, Dugas B, Hirsch EC (1999) Fc $\in$ RII/CD23 is expressed in Parkinson's disease and induces, in vitro, production of nitric oxide and tumor necrosis factor- $\alpha$ in glial cells. J Neurosci 19:3440-3447.

Janelsins MC, Mastrangelo MA, Oddo S, LaFerla FM, Federoff HJ, Bowers WJ (2005) Early correlation of microglial activation with enhanced tumor necrosis factor-alpha and monocyte chemoattractant protein-1 expression specifically within the entorhinal cortex of triple transgenic Alzheimer's disease mice. J Neuroinflammation 2:23.

Keicho N, Sawada S, Kitamura K, Yotsumoto H, Takaku F (1991) Effects of an immunosuppressant, FK506, on interleukin 1 alpha production by human macrophages and a macrophage-like cell line, U937. Cell Immunol 132:285-294.

Kim RH, Smith PD, Aleyasin H, Hayley S, Mount MP, Pownall S, Wakeham A, You-Ten AJ, Kalia SK, Horne P, Westaway D, Lozano AM, Anisman H, Park DS, Mak TW (2005) Hypersensitivity of DJ-1-deficient mice to 1-methyl-4-phenyl-1,2,3,6-tetrahydropyrindine (MPTP) and oxidative stress. Proc Natl Acad Sci USA 102:5215-5220.

Kirik D, Rosenblad C, Bjorklund A (1998) Characterization of behavioral and neurodegenerative changes following partial lesions of the nigrostriatal dopamine system induced by intrastriatal 6-hydroxydopamine in the rat. Exp Neurol 152:259-277.

Kitazawa M, Oddo S, Yamasaki TR, Green KN, LaFerla FM (2005) Lipopolysaccharide-induced inflammation exacerbates tau pathology by a cyclin-dependent kinase 5-mediated pathway in a transgenic model of Alzheimer's disease. J Neurosci 25:8843-8853.

Leng A, Mura A, Feldon J, Ferger B (2005) Tumor necrosis factor-alpha receptor ablation in a chronic MPTP mouse model of Parkinson's disease. Neurosci Lett 375:107-111.

Liu B, Du L, Hong JS (2000) Naloxone protects rat dopaminergic neurons against inflammatory damage through inhibition of microglia activation and superoxide generation. J Pharmacol Exp Ther 293:607-617.

Mabjeesh NJ, Frese M, Rauen T, Jeserich G, Kanner BI (1992) Neuronal and glial gamma-aminobutyric acid + transporters are distinct proteins. FEBS Lett 299:99-102.

MacEwan DJ (2002) TNF receptor subtype signalling: differences and cellular consequences. Cell Signal 14:477-492.

Marchetti L, Klein M, Schlett K, Pfizenmaier K, Eisel UL (2004) Tumor necrosis factor (TNF)-mediated neuroprotection against glutamateinduced excitotoxicity is enhanced by $N$-methyl-D-aspartate receptor activation. Essential role of a TNF receptor 2-mediated phosphatidylinositol 3-kinase-dependent NF-kappa B pathway. J Biol Chem 279:32869-32881.

McGeer PL, Itagaki S, Boyes BE, McGeer EG (1988) Reactive microglia are positive for HLA-DR in the substantia nigra of Parkinson's and Alzheimer's disease brains. Neurology 38:1285-1291.

McGuire SO, Ling ZD, Lipton JW, Sortwell CE, Collier TJ, Carvey PM (2001) Tumor necrosis factor alpha is toxic to embryonic mesencephalic dopamine neurons. Exp Neurol 169:219-230.

Mitoma H, Horiuchi T, Hatta N, Tsukamoto H, Harashima S, Kikuchi Y, Otsuka J, Okamura S, Fujita S, Harada M (2005) Infliximab induces potent anti-inflammatory responses by outside-to-inside signals through transmembrane TNF-alpha. Gastroenterology 128:376-392.

Mogi M, Togari A, Tanaka K, Ogawa N, Ichinose H, Nagatsu T (1999) Increase in level of tumor necrosis factor (TNF)-alpha in 6-hydroxydopamine-lesioned striatum in rats without influence of systemic L-DOPA on the TNF-alpha induction. Neurosci Lett 268:101-104.

Mogi M, Togari A, Kondo T, Mizuno Y, Komure O, Kuno S, Ichinose H, Nagatsu T (2000) Caspase activities and tumor necrosis factor receptor R1 (p55) level are elevated in the substantia nigra from parkinsonian brain. J Neural Transm 107:335-341.

Moore DJ, West AB, Dawson VL, Dawson TM (2005) Molecular pathophysiology of Parkinson's disease. Annu Rev Neurosci 28:57-87.

Nagatsu T, Sawada M (2005) Inflammatory process in Parkinson's disease: role for cytokines. Curr Pharm Des 11:999-1016.

Nishimura M, Mizuta I, Mizuta E, Yamasaki S, Ohta M, Kaji R, Kuno S (2001) Tumor necrosis factor gene polymorphisms in patients with sporadic Parkinson's disease. Neurosci Lett 311:1-4.

Oddo S, Caccamo A, Shepherd JD, Murphy MP, Golde TE, Kayed R, Metherate R, Mattson MP, Akbari Y, LaFerla FM (2003) Triple-transgenic model of Alzheimer's disease with plaques and tangles: intracellular Abeta and synaptic dysfunction. Neuron 39:409-421.

Olleros ML, Guler R, Vesin D, Parapanov R, Marchal G, Martinez-Soria E, Corazza N, Pache JC, Mueller C, Garcia I (2005) Contribution of transmembrane tumor necrosis factor to host defense against Mycobacterium bovis bacillus Calmette-Guerin and Mycobacterium tuberculosis infections. Am J Pathol 166:1109-1120.

Pasparakis M, Alexopoulou L, Episkopou V, Kollias G (1996) Immune and inflammatory responses in TNF alpha-deficient mice: a critical requirement for TNF alpha in the formation of primary B cell follicles, follicular dendritic cell networks and germinal centers, and in the maturation of the humoral immune response. J Exp Med 184:1397-1411.

Paxinos G, Watson C, Pennisi M, Topple A (1985) Bregma, lambda and the interaural midpoint in stereotaxic surgery with rats of different sex, strain and weight. J Neurosci Methods 13:139-143.

Pejovic V, Soskic V, Pan W, Kastin AJ (2004) Brain proteome of mice lacking the receptors for tumor necrosis factor alpha. Proteomics 4:1461-1464.

Przedborski S (2005) Pathogenesis of nigral cell death in Parkinson's disease. Parkinsonism Relat Disord 11 [Suppl 1]:S3-S7.

Przedborski S, Ischiropoulos H (2005) Reactive oxygen and nitrogen species: weapons of neuronal destruction in models of Parkinson's disease. Antioxid Redox Signal 7:685-693.

Ritter U, Meissner A, Ott J, Korner H (2003) Analysis of the maturation process of dendritic cells deficient for TNF and lymphotoxin-alpha reveals an essential role for TNF. J Leukoc Biol 74:216-222. 
Rousselet E, Callebert J, Parain K, Joubert C, Hunot S, Hartmann A, Jacque C, Perez-Diaz F, Cohen-Salmon C, Launay JM, Hirsch EC (2002) Role of TNF-alpha receptors in mice intoxicated with the parkinsonian toxin MPTP. Exp Neurol 177:183-192.

Sanchez-Pernaute R, Ferree A, Cooper O, Yu M, Brownell AL, Isacson O (2004) Selective COX-2 inhibition prevents progressive dopamine neuron degeneration in a rat model of Parkinson's disease. J Neuroinflammation $1: 6$

Scallon B, Cai A, Solowski N, Rosenberg A, Song XY, Shealy D, Wagner C (2002) Binding and functional comparisons of two types of tumor necrosis factor antagonists. J Pharmacol Exp Ther 301:418-426.

Sriram K, Matheson JM, Benkovic SA, Miller DB, Luster MI, O'Callaghan JP (2002) Mice deficient in TNF receptors are protected against dopaminergic neurotoxicity: implications for Parkinson's disease. FASEB J 16:1474-1476.

Sriram K, Matheson JM, Benkovic SA, Miller DB, Luster MI, O’Callaghan JP (2006) Deficiency of TNF receptors suppresses microglial activation and alters the susceptibility of brain regions to MPTP-induced neurotoxicity: role of TNF-alpha. FASEB J 20:670-682.
Steed PM, Tansey MG, Zalevsky J, Zhukovsky EA, Desjarlais JR, Szymkowski DE, Abbott C, Carmichael D, Chan C, Cherry L, Cheung P, Chirino AJ, Chung HH, Doberstein SK, Eivazi A, Filikov AV, Gao SX, Hubert RS, Hwang M, Hyun L, et al. (2003) Inactivation of TNF signaling by rationally designed dominant-negative TNF variants. Science 301:1895-1898.

Sukal SA, Nadiminti L, Granstein RD (2006) Etanercept and demyelinating disease in a patient with psoriasis. J Am Acad Dermatol 54:160-164.

Takeshima T, Johnston JM, Commissiong JW (1994) Mesencephalic type 1 astrocytes rescue dopaminergic neurons from death induced by serum deprivation. J Neurosci 14:4769-4779.

Tartaglia LA, Rothe M, Hu YF, Goeddel DV (1993) Tumor necrosis factor's cytotoxic activity is signaled by the p55 TNF receptor. Cell 73:213-216.

Vawter MP, Dillon-Carter O, Tourtellotte WW, Carvey P, Freed WJ (1996) TGFbeta1 and TGFbeta2 concentrations are elevated in Parkinson's disease in ventricular cerebrospinal fluid. Exp Neurol 142:313-322.

West MJ, Slomianka L, Gundersen HJ (1991) Unbiased stereological estimation of the total number of neurons in the subdivisions of the rat hippocampus using the optical fractionator. Anat Rec 231:482-497. 